\title{
DESENVOLVIMENTO DE UM SISTEMA FOTOVOLTAICO AUTOMÁTICO DE CARREGAMENTO DE BATERIAS PARA UMA EMBARCAÇÃO TELEOPERADA
}

Aline Damm Falcão Martins - alinedamm@gmail.com

Universidade do Estado do Rio de Janeiro

Rua São Francisco Xavier, $n^{\circ} 524$, sala 5001E

20550-900 - Rio de Janeiro - RJ

Maria Dias Bellar-bellar@ieee.org

Universidade do Estado do Rio de Janeiro

Rua São Francisco Xavier, $n^{\circ} 524$, sala 5001E

20550-900 - Rio de Janeiro - RJ

José Paulo Vilela Soares da Cunha-jpaulo@ieee.org

Universidade do Estado do Rio de Janeiro

Rua São Francisco Xavier, $n^{\circ} 524$, sala 5001E

20550-900 - Rio de Janeiro - RJ

Caio Müller-muller.caio@engenharia.ufjf.br

Universidade Federal do Rio de Janeiro

Rua Horácio Macedo, Bloco H

219471-972 - Rio de Janeiro - RJ

Resumo: O projeto de uma embarcação teleoperada motivou diversos subprojetos em um curso de graduação em engenharia elétrica, envolvendo alunos das ênfases Sistemas Eletrônicos, Sistemas de Potência, Sistemas de Telecomunicações e Sistemas e Computação. Dentro desse contexto, neste trabalho foi desenvolvido o estudo de painéis fotovoltaicos e foi feita a conexão destes para fornecer energia a baterias em um barco teleoperado. Visando conectar dois painéis fotovoltaicos a duas baterias e aos demais circuitos elétricos do barco, foram desenvolvidos circuitos eletrônicos compostos por: sensores de tensão, sensores de corrente, sensor de temperatura e acionadores de relés com componentes disponíveis e de fácil acesso no mercado. Também foi desenvolvido um circuito de acionamento de relés, com o objetivo de conectar ou desconectar partes dos circuitos e painéis quando necessário. Os relés são acionados por saídas digitais do microcontrolador Arduino. Para avaliação do sistema completo, os testes foram realizados com uma bateria e um painel fotovoltaico, conectados aos sensores e circuitos de acionamento de relés. Concluiu-se que, a bateria é carregada satisfatoriamente com a energia do painel fotovoltaico. Esse projeto pode ser reproduzido no meio acadêmico por estudantes e pesquisadores viabilizando um aprendizado em energia renovável e carregamento de baterias com baixo custo e simplicidade.

Palavras- chave: Painéis Fotovoltaicos, Bateria, Embarcação Teleoperada, Sensores, Microcontrolador. 


\section{INTRODUÇÃO}

O ensino da engenharia baseado em projetos conduz os alunos a analisarem problemas reais. Dessa forma, são definidos objetivos que precisam ser atingidos considerando as limitações técnicas e econômicas.

Com o avanço tecnológico e científico, os problemas ambientais estão cada vez mais se tornando preocupações em escala global. Monitorar fenômenos da natureza e coletar dados tem se tornado muito importante na última década. Por isso, veículos autônomos com o objetivo de observar os mares e rios estão sendo requisitados e pesquisados (CRUZ, 2008).

$\mathrm{Na}$ Universidade do Estado do Rio de Janeiro, o projeto de um veículo aquático remotamente controlado foi inicialmente discutido por (SCHULTZE, 2012), e por ser um projeto multidisciplinar, veio a dar origem a diversos outros projetos científicos nessa universidade, envolvendo energia e eletrônica de potência (PEREIRA, 2013), controle automático (ARRUDA, 2012), sensores e instrumentação (ROSARIO, 2013), propulsão (SOUZA, 2016) e comunicação (PINTO, et al, 2015), tendo-se em vista o desenvolvimento de embarcações não tripuladas.

A partir desses trabalhos conclui-se que a geração de energia elétrica na embarcação é essencial para que o projeto seja desenvolvido. Sabe-se que o sol é uma fonte inesgotável de energia renovável, com isso o acoplamento de painéis fotovoltaicos nesse veículo se torna um elemento chave para converter luz solar em eletricidade.

A motivação do projeto de graduação de (FALCÃO, 2018) foi a utilização de painéis fotovoltaicos para fornecer energia em embarcações não tripuladas. Neste trabalho, os resultados de testes realizados com painéis fotovoltaicos foram analisados para permitir o projeto e garantir o bom desempenho do sistema. Para atingir esse objetivo, foram desenvolvidos circuitos elétricos e eletrônicos simples para serem acoplados ao microcontrolador, e possibilitar o carregamento das baterias pelos painéis fotovoltaicos. Para avaliar a funcionalidade operacional do sistema, foram feitos testes do sistema acoplado a um painel fotovoltaico e a uma bateria.

$\mathrm{Na}$ literatura encontram-se trabalhos de carregamento de baterias utilizando painéis fotovoltaicos, a utilização de microcontrolador para comandar a lógica de acionamento, como os trabalhos de (SANTAMARIA et al, 2018); (GALINDO et al, 2019). Neste contexto, a proposta deste trabalho é o desenvolvimento de um sistema de carregamento de baterias em um barco teleoperado utilizando, para este fim, componentes de baixo custo e fácil aquisição (commercial off-the-shelf), o que viabiliza a sua execução na realidade universitária brasileira.

O presente trabalho está organizado da seguinte forma: na Seção 2 são apresentados o painel fotovoltaico e a bateria; na Seção 3 os componentes eletrônicos foram conectados no circuito para serem utilizados em condições marítimas; na Seção 4 é apresentada a lógica do microcontrolador para comandar o sistema; na Seção 5 foram realizados testes com um painel fotovoltaico e com uma bateria conectados ao circuito eletrônico completo; por fim, na Seção 6 são apresentadas as conclusões do trabalho.

\section{ESCOLHA DO PAINEL FOTOVOLTAICO E DA BATERIA}

A embarcação teleoperada de pequeno porte desenvolvida nos projetos de graduação citados possui casco duplo (catamarã), motores elétricos, uma central eletrônica para o controle do barco e comunicação, e um sistema de armazenamento de energia composto por baterias. O casco duplo permite a instalação de até seis painéis fotovoltaicos, como na Figura 1, usados como fonte de energia para alimentar os componentes e recarregar as baterias, aumentando o seu tempo de operação consideravelmente. 
C. COBENCE

"Os desafios para formar hoje o engenheiro do amanhã"
$\mathrm{Ol} \mathrm{a} 03$ de dezembro Evento On-line

Figura 1 - Esboço do catamarã.

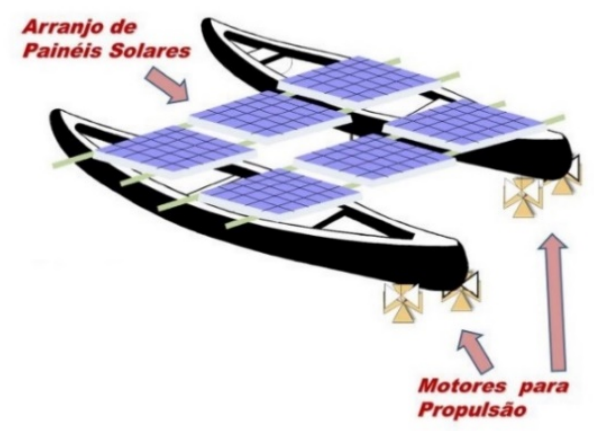

Fonte: Autor

\subsection{Painéis fotovoltaicos da embarcação}

O painel fotovoltaico escolhido foi o Kyocera KD140SX. Foram feitos alguns testes simples em dias ensolarados e nublados, também com carga variada para medir as características do painel escolhido. O circuito elétrico reproduzível utilizado para essa medição é composto por um painel fotovoltaico, um amperímetro, dois reostatos e um voltímetro, conectados conforme a Figura 2. A chave fechada permite que o circuito seja testado apenas com o resistor de $11 \mathrm{ohms}$ que suporta maior corrente (até $8 \mathrm{~A}$ ), e aberta permite que os reostatos fiquem em série para as medições com menor corrente.

Figura 2 - Circuito para medir as características do painel fotovoltaico.

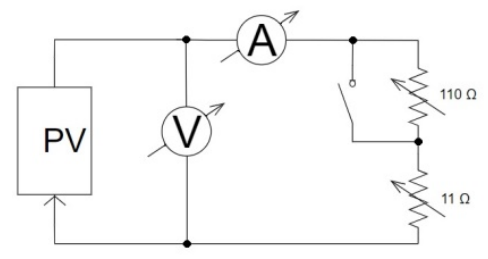

Fonte: Autor

As características elétricas obtidas nos testes em um dia ensolarado podem ser observadas nos gráficos da Figura 3, sendo o gráfico $3(\mathrm{a})$ a curva $\mathrm{I} \times \mathrm{V}$ e o gráfico $3(\mathrm{~b})$ a curva $\mathrm{P} \times \mathrm{V}$ do painel fotovoltaico.

Figura 3 - Características elétricas do painel fotovoltaico.

(a)

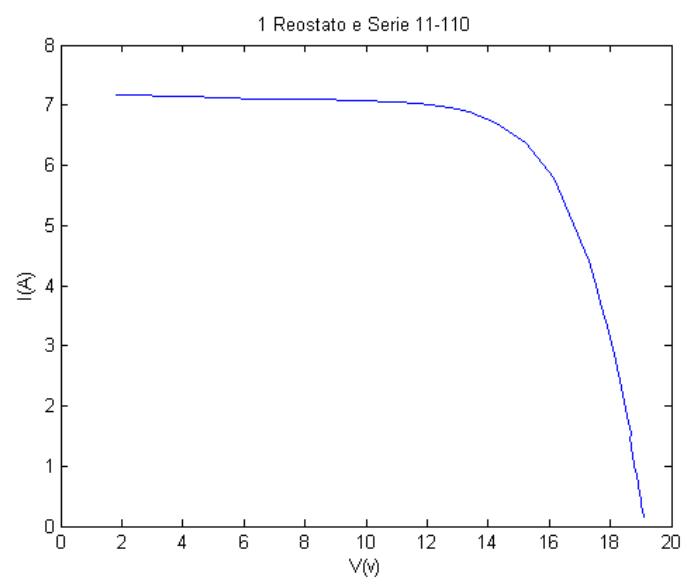

(b)

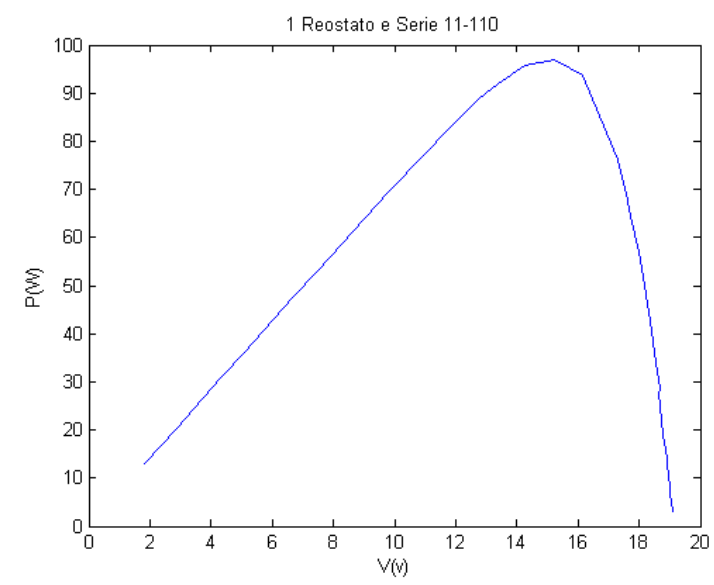

Fonte: Autor 
As Figuras 3 (a) e (b) representam um dia quente e sem nuvens. Ao decorrer da maior parte do experimento, a corrente se mantém constante em $7 \mathrm{~A}$ e a partir de $14 \mathrm{~V}$ a corrente cai até chegar a $0 \mathrm{~A}$. O pico de potência chega a $98 \mathrm{~W}$. A comparação desses valores é fundamental para se poder realizar estimativas das quantidades de potência e energia geradas, bem como verificar a compatibilidade de ligação com outros componentes do sistema fotovoltaico.

A variação de temperatura foi analisada e ela permite o estudo do funcionamento do painel com mais detalhes. A variação de temperatura do painel foi medida de $10 \mathrm{em} 10$ segundos, com o uso de um sensor (Seção 3.1), sendo que o início da contagem foi imediatamente após o painel ser colocado ao sol. A medição se inicia com uma temperatura de $24^{\circ} \mathrm{C}$ e vai gradativamente aumentando até atingir uma temperatura ao redor de $37^{\circ} \mathrm{C}$. Após 10 minutos de exposição ao sol a temperatura do painel converge para o seu valor final.

Medir as características térmicas é importante pois o aumento da temperatura nas células que compõem o módulo fotovoltaico, e que pode chegar aos $70^{\circ} \mathrm{C}$, em geral resulta em redução do seu rendimento (LANDIN, 2010, p.24).

\subsection{Bateria utilizada}

A escolha foi baseada em uma comparação com outras baterias disponíveis no mercado brasileiro que teriam a capacidade de suportar descargas e recargas contínuas. Com a finalidade de transformar energia química em energia elétrica e vice-versa, a bateria chumboácido é capaz de ser carregada e descarregada por centenas de vezes.

A bateria marine escolhida foi a bateria Moura Boat modelo 12MB105. A escolha foi baseada na disponibilidade no mercado local, o preço e a capacidade de suportar descargas e recargas profundas. $\mathrm{O}$ termo descarga se refere ao momento em que a bateria fornece energia elétrica. $\mathrm{O}$ termo carga se refere à condição na qual a bateria armazena a energia elétrica em forma de energia química (BASTOS, 2013).

A operação de uma bateria, usada em um sistema com painéis fotovoltaico, deve atender a dois tipos de ciclos fundamentais. Os ciclos rasos em dias ensolarados, que são descargas rápidas com elevadas taxas de corrente e reduzida profundidade de descarga, e ciclos profundos para tempo nublado que é utilizada quando é necessário fazer repetidos ciclos de carga e descarga completos (FADIGAS, 2012). A aplicação para esse trabalho requer baterias de ciclo profundo devido a possíveis variações climáticas durante o experimento.

\section{SISTEMA FOTOVOLTAICO PARA CARGA DE BATERIA}

Para o desenvolvimento do projeto, é necessário medir a corrente e a tensão fornecida pelos painéis fotovoltaicos. Após estudar as necessidades e as reais características de funcionamento do painel fotovoltaico, foram realizados circuitos de medição e de controle para conectá-lo ao barco. Neste sistema estão presentes sensores de temperatura, corrente, de tensão, e circuitos de acionamento de relés. Na escolha dos componentes foram consideradas as especificações do projeto, bem como o preço e a disponibilidade no mercado.

$\mathrm{Na}$ Figura 4, é apresentado um diagrama de bloco do sistema desenvolvido. O relé, que é acionado por saídas digitais do Arduino, faz a conexão e desconexão do painel fotovoltaico à bateria. O barramento $\mathrm{CC}(+12 \mathrm{~V})$ alimenta a carga. O sensor de tensão é utilizado em conjunto com o Arduino Mega através de um programa de gerenciamento de carga, onde o controlador de carga deve ligar o carregamento quando a bateria alcança o seu nível mínimo especificado de tensão e, desconectar o sistema do painel fotovoltaico quando a bateria atinge o nível máximo de tensão permitido. 
Além disso, quando a tensão do barramento, o qual é conectado em paralelo com a bateria, obtém um valor superior à tensão máxima estabelecida $(14,5 \mathrm{~V})$, o circuito é fechado. Para o circuito ser acionado existem duas condições conjuntas, a primeira é que a tensão do painel seja superior à tensão do barramento e a segunda é que a tensão do barramento seja inferior à tensão mínima estabelecida $(12 \mathrm{~V})$. O Sensor de corrente é utilizado como outra opção para fechamento do circuito, caso a corrente do painel seja inferior a corrente mínima estabelecida (0,2 A). Nas próximas Seções as partes estão descritas.

Figura 4 - Diagrama de blocos do sistema fotovoltaico desenvolvido.

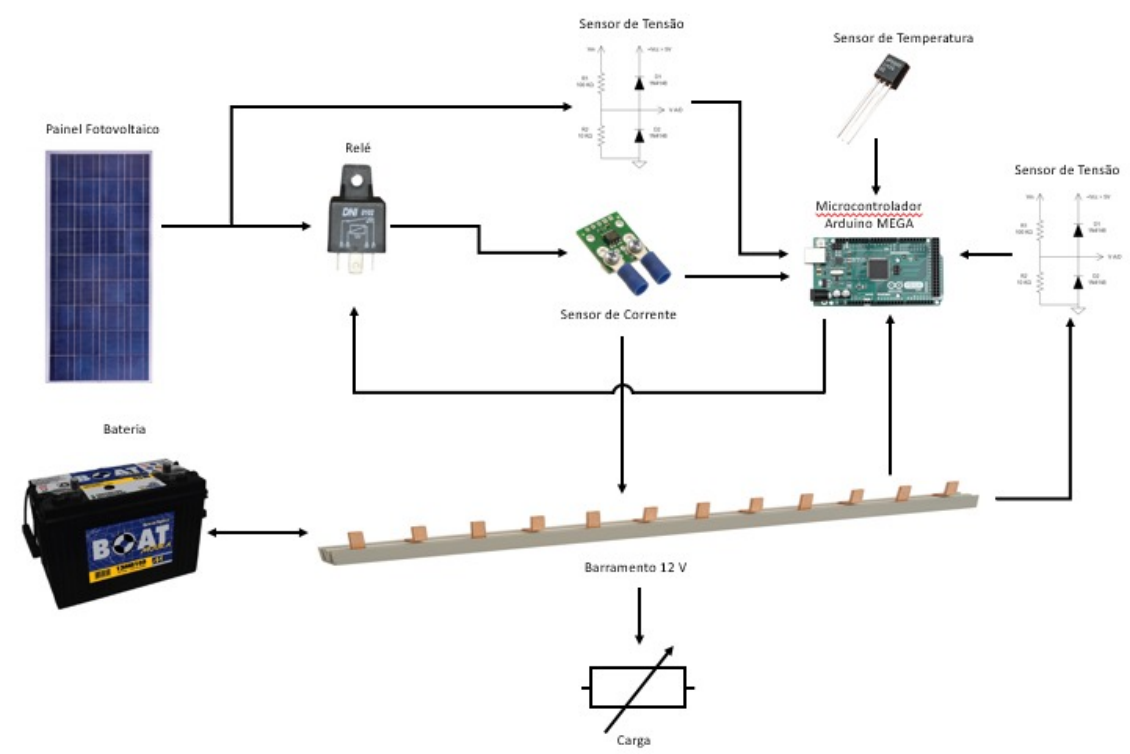

Fonte: Autor

\subsection{Sensor de temperatura}

O sensor LM35 é um sensor de precisão que apresenta uma saída de tensão linear proporcional à temperatura em que ele se encontrar no momento, tendo em sua saída um sinal de $10 \mathrm{mV}$ para cada Grau Célsius de temperatura. Esse sensor não necessita de qualquer calibração externa para fornecer com exatidão, valores temperatura com variações de $1 / 4^{\circ} \mathrm{C}$ ou até mesmo $3 / 4^{\circ} \mathrm{C}$ dentro da faixa de temperatura entre $-55^{\circ} \mathrm{C}$ e $+150^{\circ} \mathrm{C}$ (TEXAS INSTRUMENTS, 2017).

\subsection{Sensor de corrente}

O sensor de corrente é o componente ACS711. Para a utilização do sensor no projeto, necessitou-se a calibração do mesmo. A calibração foi feita com o auxílio do microcontrolador Arduino UNO. É realizada de maneira contínua e constante a leitura da corrente e tensão para controlar a carga e descarga da bateria e do painel fotovoltaico.

\subsection{Sensor de tensão}

O sensor de tensão é um circuito desenvolvido simples e que pode ser reproduzido que possui um divisor de tensão resistivo que atenua o sinal de entrada (Vin) 11 vezes. Os diodos protegem as entradas dos conversores A/D contra sobretensões como é mostrado na Figura 5. Além disso, o sensor de tensão foi calibrado com o microcontrolador Arduino UNO. 
Figura 5 - Sensor de tensão.

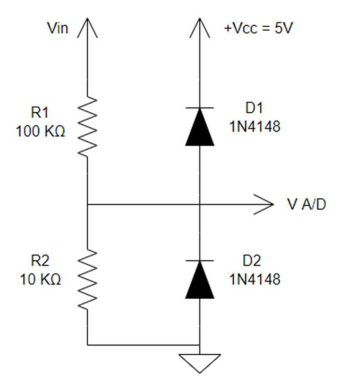

Fonte: Autor

\subsection{Relés}

Os relés serão usados para acionar o guincho da âncora, a iluminação e a conexão dos painéis fotovoltaicos às baterias. $\mathrm{O}$ circuito projetado deverá acionar relés quando necessário. Para isto foi projetado um circuito acionador de relés. A Figura 6 apresenta o circuito esquemático com os componentes selecionados onde foram feitos teste de acionamento.

A conclusão dos testes foi que, com uma alta corrente, o relé se magnetiza e fecha os contatos, e com uma determinada baixa corrente o relé mantém o estado, porém com menor consumo de potência. O relé só abre novamente com uma corrente consideravelmente menor do que a de magnetização para fechar.

Figura 6 - Circuito de acionamento do relé.

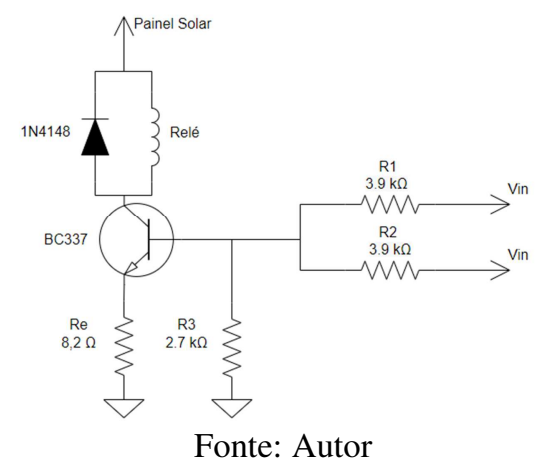

\subsection{Integração dos Circuitos}

Foi desenvolvida uma placa de interface do microcontrolador Arduino com os sensores e relés. Foi adicionado um espaço para serem dispostos três sensores de tensão, três de corrente, seis relés e dois sensores de temperatura. Ao total foram conectados dois painéis fotovoltaicos Kyocera KD 140SX, duas baterias modelo 12MB 105 de 12 V no sistema. Fusíveis de 10 A instalados nas entradas dos painéis fotovoltaicos e relés DNI 0102 estão em série nessa entrada. Esses componentes são conectados à placa eletrônica, onde há um controle realizado pelo microcontrolador Arduino.

A montagem de uma caixa de estanque para acoplar o sistema foi feita a partir dos diagramas previamente desenvolvidos. Foi estudado o posicionamento de cada elemento para maior aproveitamento e organização do espaço dentro da caixa. Foi separado um espaço disponível para futuras adições de componentes, caso necessário.

Para testar a configuração foram utilizados uma bateria, um painel fotovoltaico, um relé e os componentes agora inseridos na placa de circuito impresso, que são: o circuito acionador 
do relé, dois sensores de tensão, um sensor de corrente e o Arduino Mega. Na Figura 7 é apresentada a caixa completa, sendo (a) o diagrama das conexões para o teste e (b) o sistema físico com cabeamento definido de acordo com tamanho e cores estabelecidas para melhor reconhecimento e manutenção. O barramento e as caixas de caixas de fusíveis estão instalados com indicação de cores e numeração, respectivamente.

(a)

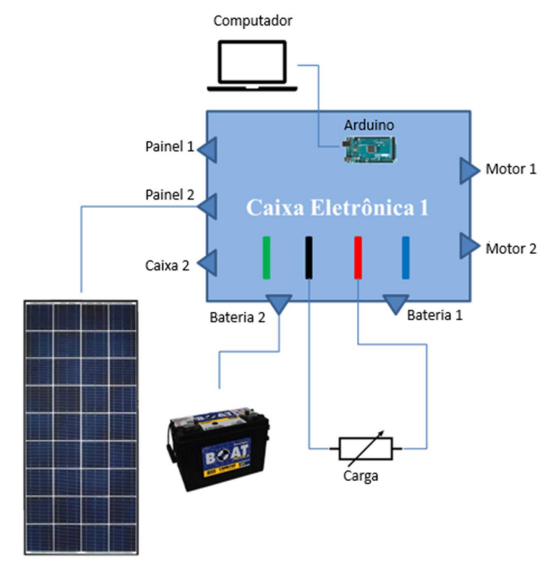

(b)

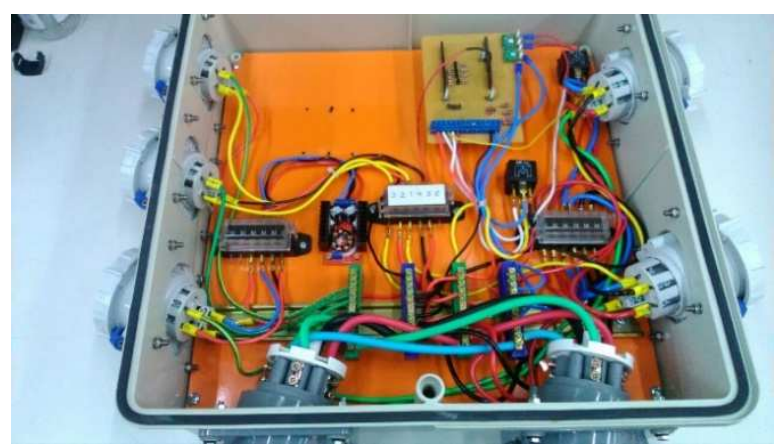

Fonte: Autor

\section{LÓGICA DO CONTROLE DE CARGA}

Para programar o gerenciamento de carga da bateria, o controlador de carga liga o carregamento quando a bateria alcança o seu nível mínimo especificado de tensão e, desconecta o sistema do painel fotovoltaico quando a bateria atinge o nível máximo de tensão permitido. Para este trabalho utiliza-se o Arduino como controlador de carga, e onde a estratégia de controle está baseada em avaliar a tensão instantânea nos terminais da bateria e comparar aos limites superior e inferior.

Uma bateria com funcionamento apropriado apresentará tensão estável e, no máximo, $10 \%$ inferior à tensão de uma bateria nova. Já uma bateria descarregada é identificada quando a queda de tensão apresentada é superior aos $10 \%$. A tensão individual de cada bateria não deverá exceder $16 \mathrm{~V}$ e quanto menos por cento de sua capacidade total ela for descarregada a sua durabilidade se estende.

Foram criadas e definidas duas funções de código bases para o sistema, para leitura da corrente e para leitura da tensão. O programa se inicializa e então é feita a leitura das variáveis. Se a tensão do barramento for maior que a tensão máxima, o painel é desconectado da bateria. Se a corrente do painel for menor que a corrente mínima, o painel também é desconectado da bateria. Se a tensão do painel for maior do que a tensão do barramento e a tensão do barramento forem menores que a tensão mínima, o painel é conectado na bateria.

\section{RESULTADOS E DISCUSSÃO}

Foram realizados testes em dia de sol com nuvens, nublado e ensolarado para validação do experimento. Durante o verão a incidência solar no local do teste se inicia após o meio dia. Neste intervalo de tempo o microcontrolador colheu dados com periodicidade de 1 segundo.

A Figura 8 apresenta os gráficos dos resultados de carregamento da bateria (a), corrente no painel (b) e tensão na bateria (c) para o dia ensolarado. É possível notar que a bateria foi 


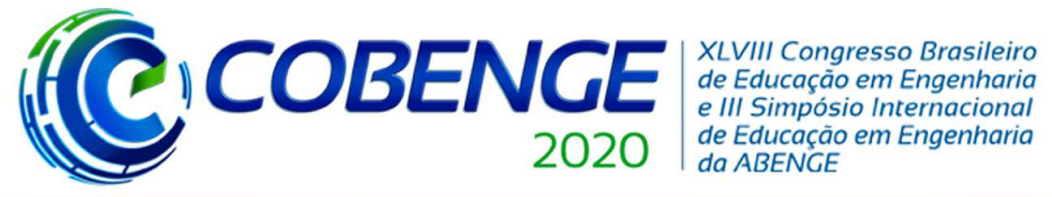

"Os desafios para formar hoje o engenheiro do amanhã"
O1 a 03 de dezembro Evento On-line

carregada de maneira efetiva. Além disso, 40 minutos após o início do experimento, o painel que fora iniciado na horizontal, foi inclinado em $30^{\circ}$, essa mudança é perceptível no gráfico da Figura 8(b) onde há um aumento de $1 \mathrm{~A}$. O painel é rotacionado mais uma vez, horizontalmente na direção do sol no tempo de 3:30 horas após o início do experimento onde há outra variação perceptível na corrente.

A temperatura medida nos experimentos variava entre $30^{\circ} \mathrm{C}$ e $42^{\circ} \mathrm{C}$. Assim, a condição de manter o painel abaixo de $70^{\circ} \mathrm{C}$ como recomenda o fabricante foi satisfeita.

Figura 8 - Resultados experimentais de carregamento da bateria.

(a)

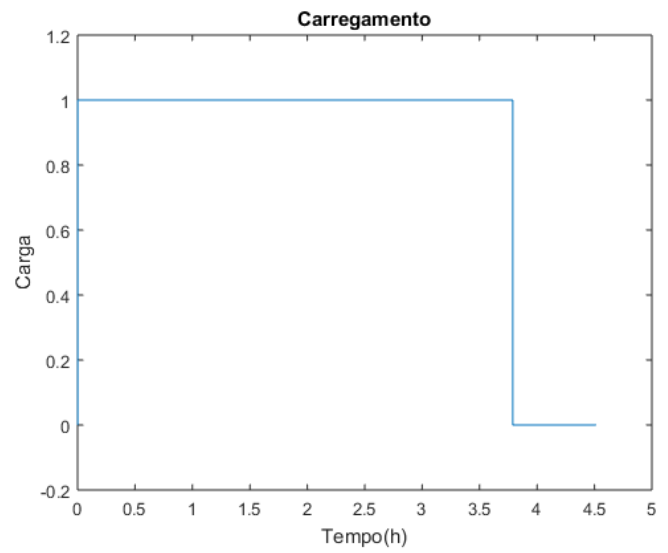

(c)

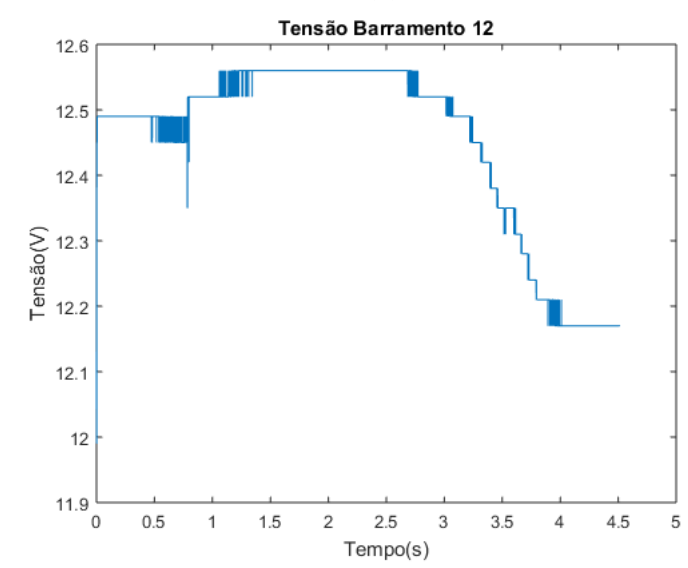

(b)

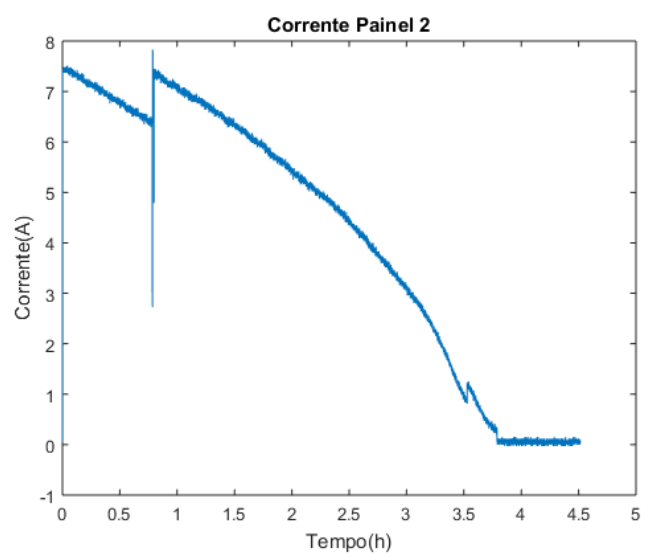

Fonte: Autor

\section{CONCLUSÃO}

Esse trabalho descreveu um projeto de graduação sobre aplicação de painéis fotovoltaicos para fornecimento de energia elétrica em uma embarcação teleoperada. O desenvolvimento dessa embarcação tem motivado diversos projetos de graduação em engenharia elétrica. Com isso, em vez de desenvolver diversos pequenos projetos de graduação isolados, a ideia aqui é desenvolver um grande projeto á longo prazo que motive a realização de projetos de graduação interligados.

Neste trabalho foi utilizada uma metodologia de ensino de engenharia baseada em projetos. O presente projeto tem as seguintes etapas: a proposição do problema, a seleção e modelagem de partes do sistema, a concepção do sistema, o desenvolvimento das partes, sua integração e, por fim, o teste do conjunto.

Os experimentos e análises iniciais sobre as características do painel fotovoltaico possibilitaram a etapa seguinte que foi construir um circuito que conecte o painel a uma 
bateria. Durante o desenvolvimento deste projeto foi realizada a construção e a calibração de sensores de tensão e de corrente e a elaboração de circuitos de acionamento com relés. Os circuitos desenvolvidos bem como a eletrônica aplicada a eles se mostraram eficazes.

Os resultados obtidos indicaram que o painel fotovoltaico foi capaz de carregar a bateria de forma automática. Uma próxima etapa será a sua integração e teste no catamarã.

Esse projeto demonstra que existem meios simples e de baixo custo para estudar o funcionamento de um painel fotovoltaico com fins educacionais e controlá-lo para o fornecimento de energia em conjunto com baterias.

\section{Agradecimentos}

À Universidade do Estado do Rio de Janeiro - Campus Maracanã. À Faperj e ao CNPq, que financiaram parte deste trabalho.

\section{REFERÊNCIAS}

ARRUDA, José Wilton Oliveira de. Controle Automático de Rumo de uma Embarcação de Superfície não Tripulada. 2012. 96 f. Trabalho de Conclusão de Curso (Graduação) Engenharia Elétrica, Universidade do Estado do Rio de Janeiro, Rio de Janeiro, 2012.

BASTOS, Renan Fernandes. Sistema de gerenciamento para carga e descarga de baterias. 2013. 115 f. Dissertação (Mestrado) - Engenharia Elétrica, Universidade de São Paulo, São Carlos, 2013.

CRUZ, N.A.; ALVES, J. C. Autonomous sailboats: An emerging technology for ocean sampling and surveillance. IEEE 2008 OCEANS Conference, OCEANS'08, Quebec City, Canada, pp. 1-6, 2008.

FADIGAS, E. A. F. A. Energia Solar Fotovoltaica: Fundamentos, Conversão e Viabilidade técnico-econômica. Relatório do Grupo de Energia Escola Politécnica Universidade de São Paulo, São Paulo, 2012.

FALCÃO, Aline Damm da Silva. Sistema de Painéis Fotovoltaicos e Baterias para um Barco Teleoperado. 2018. 80 f. Trabalho de Conclusão de Curso (Graduação) - Engenharia Elétrica, Universidade do Estado do Rio de Janeiro, Rio de Janeiro, 2018.

GALINDO, Jonas M. S.; PLÁCIDO, José E. S.; ARAÚJO, Jackson A.; Experiências de aprendizagem baseado em projetos no ensino da graduação em engenharia elétrica. In: XLVII Congresso Brasileiro de Educação em Engenharia, 2019, Fortaleza. Anais. Fortaleza, 2019.

LANDIN, E. Energia solar fotovoltaica. Niquelândia, 2010.

PEREIRA, Mayra da Veiga Peixoto. Sistemas de Eletrônica de Potência para Barco Teleguiado Baseado em Módulos Solares Fotovoltaicos. 2013. 105 f. Trabalho de Conclusão de Curso (Graduação) - Engenharia Elétrica, Universidade do Estado do Rio de Janeiro, Rio de Janeiro, 2013. 
PINTO, Paulo Henrique Silva; Mok, Roberto Wu. Projeto de uma Embarcação Multicasco Teleoperada. 2015. 96 f. Trabalho de Conclusão de Curso (Graduação) - Engenharia Elétrica, Universidade do Estado do Rio de Janeiro, Rio de Janeiro, 2015.

ROSARIO, Rafael Vida de Castro. Sistema para Monitoração de uma Embarcação não Tripulada. 2013. 66 f. Trabalho de Conclusão de Curso (Graduação) - Engenharia Elétrica, Universidade do Estado do Rio de Janeiro, Rio de Janeiro, 2013.

SANTAMARIA, M.; CORREA, N. M.; MORENO, J. E.; MORENO, Y. E. Proposal Design of an Open Source Automated System for Photovoltaic Panels Characterization by Electronic Charge Method. IEEE Latin America Transactions, vol. 16, no. 6, pp. 1644-1649, 2018.

SCHULTZE, Hendrik Jürgen. Projeto e Construção de uma Embarcação Teleoperada. 2012. 84 f. Trabalho de Conclusão de Curso (Graduação) - Engenharia Elétrica, Universidade do Estado do Rio de Janeiro, Rio de Janeiro, 2012.

SOUZA, Lenielson Rodrigues de. Acionamento dos Motores CC de uma Embarcação Teleoperada. 2016. 127 f. Trabalho de Conclusão de Curso (Graduação) - Engenharia Elétrica, Universidade do Estado do Rio de Janeiro, Rio de Janeiro, 2016.

TEXAS INSTRUMENTS. LM35 Precision Centigrade Temperature Sensors. Texas, 2017.

\title{
DEVELOPMENT OF AN AUTOMATIC PHOTOVOLTAIC SYSTEM FOR BATTERY RECHARGE IN AN UNMANNED SURFACE VESSEL
}

\begin{abstract}
The design of an unmanned surface vessel (USV) motivated several subprojects in an electrical engineering undergraduate course, involving students in the fields of Electronic Systems, Power Systems, Telecommunication Systems and Computing. Within this context, in this work the study of photovoltaic modules was developed and their connection was made to supply energy to batteries in an USV. In order to connect two photovoltaic modules with two batteries and to the other electrical circuits of the USV, electronic circuits were developed, comprising: voltage sensors, current sensors, temperature sensor and relay drivers with commercial off-the-shelf (COTS) parts. A relay drive circuit was also developed, with the objective of connecting or disconnecting parts of the circuits and photovoltaic modules when necessary. The relays are driven by digital outputs of an Arduino microcontroller. To evaluate the complete system, the tests were performed with a battery and a photovoltaic module connected to the sensors and relay drive circuits. In conclusion, the battery can be recharged satisfactorily with the energy of the photovoltaic module. This project can be reproduced in the academic environment by students and researchers, enabling learning in renewable energy and recharging batteries with low cost and simplicity.
\end{abstract}

Keywords: Photovoltaic module, Battery, Unmanned surface vessel, Sensor, Microcontroller. 